\title{
Modified biophysical profile and perinatal outcome in high risk pregnancies
}

\section{Gulafshan Anjum, Hina Mittal, Nidhi Chauhan*}

Department of Obstetrics and Gynecology, Himalayan Institute, Dehradun, Uttrrakhand, India

Received: 09 April 2021

Accepted: 01 May 2021

\section{*Correspondence:}

Dr. Nidhi Chauhan,

E-mail: nidhichauhan@srhu.edu.in

Copyright: (c) the author(s), publisher and licensee Medip Academy. This is an open-access article distributed under the terms of the Creative Commons Attribution Non-Commercial License, which permits unrestricted non-commercial use, distribution, and reproduction in any medium, provided the original work is properly cited.

\section{ABSTRACT}

Background: Aim and objective of current investigation was to evaluate perinatal outcome in high risk pregnancy with modified biophysical profile and also evaluate the efficacy of MBPP.

Methods: Type of study was observational study, 100 patient fulfilling inclusion criteria were included in study. All women were subjected to modified biophysical profile comprises amniotic fluid index and non stress test. NST was performed with cardiotocogram, real time ultrasound scanning was performed. Perinatal outcome assessed in terms of admission to NICU, low birth weight, foetal distress, low APGAR score, neonatal mortality.

Results: Out of 100 cases, 55 cases had reactive NST and 45 had non reactive NST. AFI was normal in 79 cases 21 cases had abnormal AFI. Diagnostic power was maximum seen with NST i.e. $76.36 \%$ (61.90\% for AFI and 71.64\% for combined MBPP) it indicates that NST is a good predictor to diagnose a compromised foetus.

Conclusions: This study shows that pregnancy with high risk factors are associated with more chances of intrapartum complications perinatal morbidity and mortality, if MBPP was abnormal or any one parameter was abnormal, chances of perinatal morbidity were high.

Keywords: Pregnancy, Perinatal outcome, Biophysical profile

\section{INTRODUCTION}

In antenatal period, nearly $1 / 3^{\text {rd }}$ to $1 / 2^{\text {nd }}$ of the perinatal deaths occurs in most centers. The objective of antepartum surveillance is to improve the perinatal outcome through the timely diagnosis and treatment of fetal compromise and to ensure the fetal well being. A variety of option are available for use in high risk pregnancy. Modified biophysical profile is the best available test for primary fetal surveillance. ${ }^{1}$ It combines the observation of an index of acute fetal hypoxia, the NST and an indicator of chronic fetal problem amniotic fluid volume. ${ }^{2}$ MBPP is easier to perform and less time consuming than complete biophysical profile or contraction stress test. Also, MBPP is considered to be as effective as complete biophysical profile .Modified biophysical profile has excellent negative and positive predictive values of $0.8 / 1000$ and $1.5 \%$ respectively. ${ }^{3}$ NSTs are classified as reactive and non-reactive. Reactive or normal NST is characterized by 2 or more fetal heart rate accelerations of about $15 \mathrm{bpm}$ and lasting at least 15 seconds from the baseline within a 20 minute period. Non-reactive NST is characterized by lack of acceleration for a period of 40 minutes. ${ }^{4}$ The objective of "antenatal fetal monitoring to identify fetuses that are at risk so that intervention can be done before damage occurs. A reactive NST is a reliable indicator of fetal well being in term fetus". Fetal demise within 1 week of a reassuring NST defined as false negative NST which is less than $1 \% .^{5}$ Hence in this study, modified biophysical profile was used as primary surveillance test in high risk pregnancies to study its effectiveness in predicting the perinatal outcome. 


\section{METHODS}

The study was conducted in the department of obstetrics and gynecology, Himalayan institute of medical sciences (HIMS), Swami Ram Nagar, Dehradun, over a period of one year. Subjects were recruited from patients presenting in obstetric and gynecology OPD, IPD and Emergency at HIMS, Dehradun with high risk pregnancy after obtaining written informed consent and approval of ethics committee.

\section{Study design, sample size and sampling method}

Current study was an observational study conducted on 100 patients. Patients were included in the study by convenient sampling method.

\section{Inclusion criteria}

Inclusion criteria for current study were; all antenatal woman at or over 32 weeks of gestational age with preeclampsia, bad obstetric history, postdatism, intrauterine growth restriction, diabetes, Rh negative pregnancy, anemia, previous LSCS, decreased fetal movements, heart disease and asthma.

\section{Exclusion criteria}

Exclusion criteria for current study were; fetuses with congenital anomalies, antenatal women below 32 weeks of gestational age, antenatal women without risk factors, antenatal women in active labor, acute fetal distress, antenatal women with active bleeding, antenatal women with active leaking, ruptured membrane, intra uterine fetal death.

\section{Study protocol}

The complete history (name, age, sex, occupation, residential address, chief complaints, and history of presenting illness, obstetrical history, menstrual history, past history, and drug history) was taken and clinical examination was carried out. The NST was performed with cardiotocogram (BORZE.S NO.-C00035) Model no. WL 15000 in supine position. Recording of FHR, fetal movements, uterine contractions was done. The trace was considered as reactive, if more than 2 fetal movements with acceleration of more than or equal to 15 beats/minute lasting for more than or equal to 15 seconds, with good beat-to-beat variability and no decelerations. Ultrasound obstetric was performed and level of AFI was studied. Evaluation of fetal health in high risk pregnancy with the help of Modified biophysical profile was studied.

\section{Statistical analysis}

Categorical variables were presented in number and percentage $(\%)$ and continuous variables were presented as mean $\pm \mathrm{SD}$ and median. Qualitative variables were correlated using Chi-Square test/Fisher's Exact test. Odds ratio was calculated of MBPP for adverse perinatal outcome. Diagnostic test was used to find out sensitivity, specificity, NPV and PPV. A p value of $<0.05$ was considered statistically significant. The data was entered in MS Excel spreadsheet and analysis was done using statistical package for social sciences (SPSS) version 21.0 .

\section{RESULTS}

Most common high risk factor in patient included in this study was pregnancy induced hypertension $27.7 \%$, there were more than one co-morbidity present in one patient. Out of 100 high risk cases $45 \%$ patient had reactive NST and $55 \%$ had non reactive NST and 79 patients had normal AFI and 21 patients had abnormal AFI. Among patient with non reactive NST $61.81 \%$ neonate were admitted to NICU, $23.63 \%$ had foetal distress, $30.90 \%$ delivered low birth weight, $3.63 \%$ had low APGAR score, $1.81 \%$ perinatal death were seen .Patients with abnormal AFI, 52.38\% required NICU admission, $38.10 \%$ had low birth weight babies, $14.29 \%$ had foetal distress and $9.52 \%$ perinatal death, $4.76 \%$ had low APGAR score.

Table 1: Distribution of study subjects according to age group.

\begin{tabular}{|c|c|c|}
\hline Age group (years) & $\mathbf{N}$ & $\%$ \\
\hline $18-25$ & 47 & 47 \\
\hline 26-35 & 46 & 46 \\
\hline$>35$ & 7 & 7 \\
\hline
\end{tabular}

In the present study it was observed that out of the 100 high risk women $47(47 \%)$ were 18-25 years, $46(46 \%)$ were $26-35$ years, $7(7 \%)$ were $>35$ years. Most common high risk factor in patient included in this study was pregnancy induced hypertension $27.7 \%$, there were more than one co-morbidity present in one patient (Table 2).

Table 2: Distribution of study subject according to presence of high risk factors.

\begin{tabular}{|llll|}
\hline $\begin{array}{l}\text { Co- } \\
\text { morbidities }\end{array}$ & N & $\%$ \\
\hline $\begin{array}{l}\text { Anaemia } \\
\text { Hypertensive } \\
\text { disorder }\end{array}$ & Present & 11 & 7.43 \\
\hline $\begin{array}{l}\text { Gestational } \\
\text { diabetes }\end{array}$ & Present & 61 & 27.7 \\
\hline Asthma & Present & 11 & 4.05 \\
\hline Heart disease & Present & 7 & 7.43 \\
\hline Post datism & Present & 10 & 6.72 \\
\hline IUGR & Present & 25 & 16.89 \\
\hline $\begin{array}{l}\text { Previous } \\
\text { LSCS }\end{array}$ & Present & 24 & 16.21 \\
\hline $\begin{array}{l}\text { Foetal } \\
\text { movement }\end{array}$ & Reduced & 13 & 8.78 \\
\hline
\end{tabular}


Table 3: Predictability of perinatal outcome with last AFI value.

\begin{tabular}{|l|lll|l|}
\hline Parameters & Sensitivity $(\%)$ & Specificity $(\%)$ & PPV $(\%)$ & NPV (\%) \\
\hline < APGAR at 5 minutes & 50.00 & 79.59 & 4.76 & 98.73 \\
\hline Fetal distress & 15.38 & 78.16 & 9.52 & 86.08 \\
\hline Admission to NICU & 23.40 & 81.13 & 52.38 & 54.43 \\
\hline Low birth weight & 34.78 & 83.12 & 38.10 & 81.01 \\
\hline Mortality & 100 & 80.61 & 9.52 & 100 \\
\hline Combined & 22.81 & 81.40 & 61.90 & 44.30 \\
\hline
\end{tabular}

Table 4: Predictability of perinatal outcome with last NST pattern.

\begin{tabular}{|l|lll|l|}
\hline Parameters & Sensitivity $(\%)$ & Specificity $(\%)$ & PPV $(\%)$ & NPV (\%) \\
\hline < 7 APGAR at 5 minutes & 100.00 & 45.92 & 3.64 & 100.00 \\
\hline Fetal distress & 100.00 & 51.72 & 23.64 & 100.00 \\
\hline Admission to NICU & 72.34 & 60.38 & 61.82 & 71.11 \\
\hline Low birth weight & 73.91 & 50.65 & 30.91 & 86.67 \\
\hline Mortality & 50.00 & 44.90 & 1.82 & 97.78 \\
\hline Combined & 73.68 & 69.77 & 76.36 & 66.6 \\
\hline
\end{tabular}

Table 5: Predictability of perinatal outcome with modified biophysical profile.

\begin{tabular}{|llll|l|}
\hline Parameters & Sensitivity $(\%)$ & Specificity $(\%)$ & PPV $(\%)$ & NPV (\%) \\
\hline < APGAR at 5 minutes & 100.00 & 33.67 & 2.99 & 100.00 \\
\hline Fetal distress & 100.00 & 37.93 & 19.40 & 100.00 \\
\hline Admission to NICU & 80.85 & 45.28 & 56.72 & 72.73 \\
\hline Low birth weight & 95.65 & 41.56 & 32.84 & 96.97 \\
\hline Mortality & 100.00 & 33.67 & 2.99 & 100.00 \\
\hline
\end{tabular}

Overall predictability of last AFI was as sensitivity $22.81 \%$, specificity $81.40 \%$, PPV $61.90 \%$, NPV $44.30 \%$ for perinatal outcome (Table 3). Over all predictability of last NST pattern was as sensitivity $73.68 \%$, specificity $69.77 \%$, PPV $76.36 \%$, NPV $66.67 \%$ for perinatal outcome (Table 4). Total 2 neonatal mortality was seen when MBPP was abnormal with the predictable sensitivity $100 \%$, specificity $33.67 \%$, PPV $2.99 \%$, NPV $100.00 \%$ (Table 5).

\section{DISCUSSION}

In present study, study population belonged to the age group where age range varied from 18-35 years. Majority of women screened belong to $18-25$ yrs $(47 \%)$, years of age followed by age group of 26-35 (46\%) and $7 \%$ were found to be $>35$ years. In "present study the test group" consist of 100 cases of high risk pregnant females "with gestational age >32 weeks", most of them were multigravida (55\%) and $45 \%$ found to be primigravida. Among total patient, 33 cases had both parameters normal among them 24 patient $(72.73 \%)$ underwent LSCS and $9(27.27 \%)$ had vaginal delivery .Both parameter were abnormal in 9 cases and $100 \%$ patient underwent LSCS, there were no vaginal delivery. When only one parameter i.e. AFI was abnormal, $58.33 \%$ underwent LSCS and $41.67 \%$ had vaginal delivery this was corroborated by a study done by Vanamala where the mode of delivery was LSCS (44.4\%) when AFI was abnormal and when only NST was abnormal, 40 cases $(86.96 \%)$ underwent LSCS. ${ }^{6}$

Total mortality was $2(2 \%)$ in our study, both had abnormal AFI and one had reactive NST and another had non-reactive NST. So predictability of AFI in term of mortality sensitivity $100.00 \%$, specificity $80.61 \%$, positive predictive value (PPV) 9.52\%, negative predictive value (NPV) $100.00 \%$. Eden et al also found $5.94 \%$ of perinatal mortalities in their study. ${ }^{7}$ Predictability of NST in term of mortality was as sensitivity $50.00 \%$, specificity $44.90 \%$, positive predictive value (PPV) $1.82 \%$, and negative predictive value (NPV) 97.78\%. Diagnostic accuracy (sensitivity) was improved when NST and AFI both were combined. Diagnostic power (positive predictive value) was maximum seen with NST i.e. $76.36 \%(61.90 \%$ for AFI and $71.64 \%$ for combined MBPP) it indicates that NST is a good predictor to diagnose a compromised foetus.

\section{CONCLUSION}

The main objective of antepartum surveillance in high risk pregnancy to "identify the fetuses at risk of morbidity and mortality" and timely intervention to prevent the damage. MBPP is a combination of Non stress test and Amniotic fluid index which is easier, less time consuming, cost effective and patient compliant test. In this study, 100 high risk pregnant females over a 
period of one year were screened with MBPP, most of them were multigravidas. When MBPP is normal, "it gives reassurance that the fetal status is good and will have good perinatal outcome". At the same time, when MBPP is abnormal, it indicates that the fetus may be compromised and intervention is required.This study shows that pregnancy with high risk factors are associated with more chances of intrapartum complications, perinatal morbidity and mortality. If MBPP is abnormal or any one parameter is abnormal, chances of low birth weight, low APGAR score, fetal distress, NICU admission and perinatal mortality are high.

Funding: No funding sources Conflict of interest: None declared

Ethical approval: The study was approved by the Institutional Ethics Committee

\section{REFERENCES}

1. Manning FA, Harman CR, Morrison I, Menticoglou SM, Lange IR. Fetal assessment based on fetal biophysical profile scoring: IV. An analysis of perinatal morbidity and mortality. Am J Obstet Gynecol. 1990;162(3):703-9.
2. Alchalabi HA, Obeidat BR, Jallad MF, Khader YS. Induction of labor and perinatal outcome: the impact of the amniotic fluid index. Eur J Obstet Gynecol Reprod Biol. 2006;129(2):124-7.

3. Vintzileos AM, Campbell WA, Ingardia CJ, Nochimson DJ. The fetal biophysical profile and its predictive value. Obstet Gynecol. 1983;62(3):271-8.

4. Himabindu P, Tripura M, Pavani S. Evaluation of non stress test in monitoring high risk pregnancies. IOSR J Dent Med Sci. 2015;14(4):40-2.

5. Gilstrap LW. Obstetrics. 21st ed. USA: Mc Grew Hill; 2003:871.

6. Vanamala VG, Rachel A, Pakyanadhan S, Abraham SP. Biophysical profile and modified biophysical profile in predicting the fetal outcome. Int J Reprod Contracept Obstet Gynecol. 2018;7:45-9.

7. Eden RD, Seifert LS, Kodack LD, Trofatter KF, Killam AP, Gall SA. A modified biophysical profile for antenatal fetal surveillance. Obstet Gynecol. 1988;71(3):365-9.

Cite this article as: Anjum G, Mittal H, Chauhan N. Modified biophysical profile and perinatal outcome in high risk pregnancies. Int J Reprod Contracept Obstet Gynecol 2021;10:3357-60. 